\title{
Double-Pulse Remote Laser-Induced Breakdown Spectroscopy Analysis of Magnesium Alloys
}

\author{
Lifeng Qi, Lanxiang Sun*, Zhibo Cong, Yong Xin, Yang Li
}

Lab. of Networked Control Systems, Shenyang Institute of Automation, Chinese Academy of Sciences, Shenyang, China.

Email: *sunlanxiang@sia.cn

Received August 2013

\begin{abstract}
A self-built double-pulse remote Laser-Induced Breakdown Spectroscopy system in a collinear configuration was used to investigate the magnesium alloys. The enhancement of the intensity was observed, about 4.7 times compared with single pulse LIBS. The peak intensities of line Y II $366.4 \mathrm{~nm}$ and Zr I $468.7 \mathrm{~nm}$ were used in the calibration curves, and the correlation coefficients were 0.9998 and 0.9547 respectively.
\end{abstract}

Keywords: Double Pulse; LIBS; Remote; Magnesium Alloys

\section{Introduction}

The chemical elementary component analysis of metallic alloys is very important for process control and quality assessment in metallurgical processing. The dominant analytical tools applied nowadays are based on spectroscopic techniques such as inductively coupled plasma-atomic emission spectroscopy (ICP-AES), X-ray fluorescence (XRF), etc. These techniques are mostly used in off-site laboratories and require sample preparation which is time consuming. Laser-induced breakdown spectroscopy (LIBS) is a useful technique known as a spectrochemical tool for detecting the chemical composition of a wide range of materials such as metals, minerals, chemical substances, and trace species without the need of sample preparation and the analysis procedure is simple and fast [1]. It is a potential powerful technique for the metal smelting in real-time and online chemical analysisor monitoring. However, the lower sensitivity and precision than the other elemental analysis methods is one of the major drawbacks especially in remote detection [2]. To improve LIBS, a lot of approaches have been proposed to enhance the analytical performance [3-6], and the double pulse LIBS (DP-LIBS) has been demonstrated by a number of studies [7-11], which is a very efficient approach for enhancing the intensity of plasma emission and improvement of the analytical capabilities of LIBS.

In this work, a self-built remote collinear DP-LIBS is used to analyze the chemical component of magnesium alloys and compare with the single pulse LIBS about the

"Corresponding author. intensity of plasma light. The goal of this study is to confirm the effect of the intensity enhancement in remote DP-LIBS and the probability of the remote DP-LIBS technique applied in metal smelting process.

\section{Experimental}

The experimental system in this study consisted of two Nd:YAG lasers, which were characterized by a maximum repetition rate of $10 \mathrm{~Hz}$, a maximum energy of 200 $\mathrm{mJ}$ per pulse at $1064 \mathrm{~nm}$, and a full width half maximum (FWHM) of about 10 ns. A combination of half wave plate and polarization beam splitter (PBS) was used to adjustment the energy of the laser beams and alignment the two laser beams in collinear. The collinear laser beams were focused on the targets at $2.5 \mathrm{~m}$ by a combination of four lenses. The emitted plasma radiation was collected by a commercially available 12 inch Schmidt-Cassegrain telescope, then focused into an optic fiber and guided to a spectrometer (LIBS 2500) developed by Ocean Optics, Inc. The delay time between the two lasers and the gate delay time of the spectrometer was set and controlled by a versatile digital delay/pulse generator (DG645). A schematic of the experimental setup is shown in Figure 1. In order to reduce the influence of Bremsstrahlung and freebound electronic recombination continuum radiation, the gate delay time after the second laser pulse and the integrate time of the spectrometer were respectively set $3 \mu$ s and $1 \mathrm{~ms}$ in all the measurements. For all the samples, 500 spectra were acquired and averaged into a single spectrum to reduce the spectral fluctuations.

All the magnesium alloy samples in this work were 


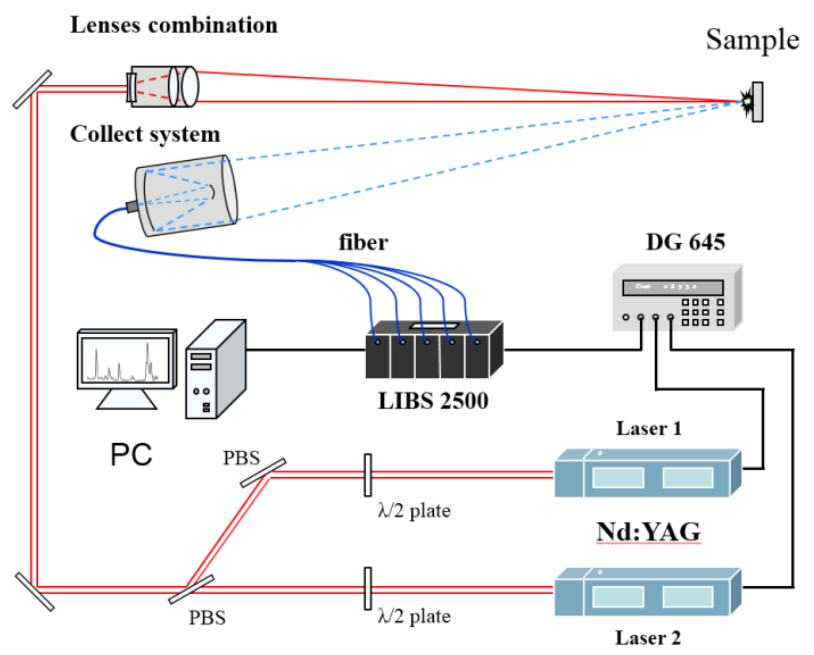

Figure 1. Theschematic of the experimental setup.

provided by the institute of metal research, Chinese academy of sciences. The average chemical compositions of the samples are listed in Table 1.

\section{Results}

The delay time between the two laser pluses plays an important role in the signal intensity of DP-LIBS [7,8,11]. Figure 2 shows the emission intensity for the $\mathrm{Zr} I$ line at $468.7 \mathrm{~nm}$ and Y II line at $366.4 \mathrm{~nm}$ of sample \#56 as a function of the delay time in DP-LIBS from 0 to $10 \mu$ s. It can be seen that the signal intensity increases by the delay time, maxima at about $6 \mu$ s. After $6 \mu$ s an almost constant intensity is observed. Consequently, the delay time between the laser pulses in DP-LIBS was fixed at 6 $\mu \mathrm{s}$ in this research.

Turned off the laser 1, just the laser 2 was on work for a single pulse LIBS in this work. Figure 3 shows significant enhancement in LIBS signal for collinear DP-LIBS compared with single pulse LIBS. The energy of the both laser pulses were fixed at $100 \mathrm{~mJ}$ in DP-LIBS, and the energy in single pulse LIBS was fixed at $200 \mathrm{~mJ}$ for keeping consistent for both single pulse and double pulse LIBS. Sample \#56 was chosen as sample for this study. Observed from the data, it can be clearly seen that the enhancement in signal intensity is about 4.7 times for collinear DP-LIBS compared with the single pulse LIBS of the Y II line at $366.4 \mathrm{~nm}$. In the remote LIBS system, the higher laser energies are required for an effective detection due to the larger propagating consumption of the laser pulses and the plasma light in the free space [12]. Using the DP-LIBS, which allows lower laser energy with enhancement of the LIBS signal is an available technique to improve the intensity and sensitivity in remote LIBS.

According to the standard concentrations of the magnesium alloy samples in Table 1, the peak intensity of line Y II $366.4 \mathrm{~nm}$ and $\mathrm{Zr}$ I $468.7 \mathrm{~nm}$ were selected for
Table 1. Average chemical compositions of the Mg-RE samples.

\begin{tabular}{cccccc}
\hline \multirow{2}{*}{$\begin{array}{c}\text { C } \\
\text { No. }\end{array}$} & \multicolumn{2}{c}{ Content } & \multicolumn{3}{c}{ w/\% } \\
\hline \multirow{2}{*}{$\# 56$} & 6.12 & 1.14 & 0.58 & & residue \\
$\# 138$ & 3.79 & 2.22 & 0.5 & & residue \\
$\# 139$ & 4.75 & 1.41 & 0.53 & & residue \\
$\# 118$ & & 2.1 & 0.32 & 9.32 & residue \\
$\# 132$ & 0.48 & 2.7 & 0.4 & 9.44 & residue \\
\hline
\end{tabular}

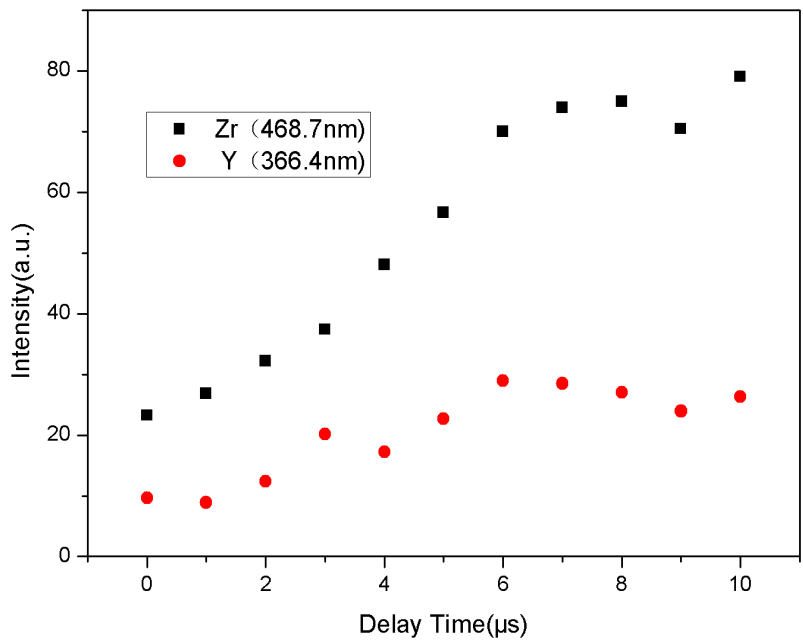

Figure 2. Intensity vs. gate delay time for the Y II $366.4 \mathrm{~nm}$ and Zr I 468.7nm (\#56).

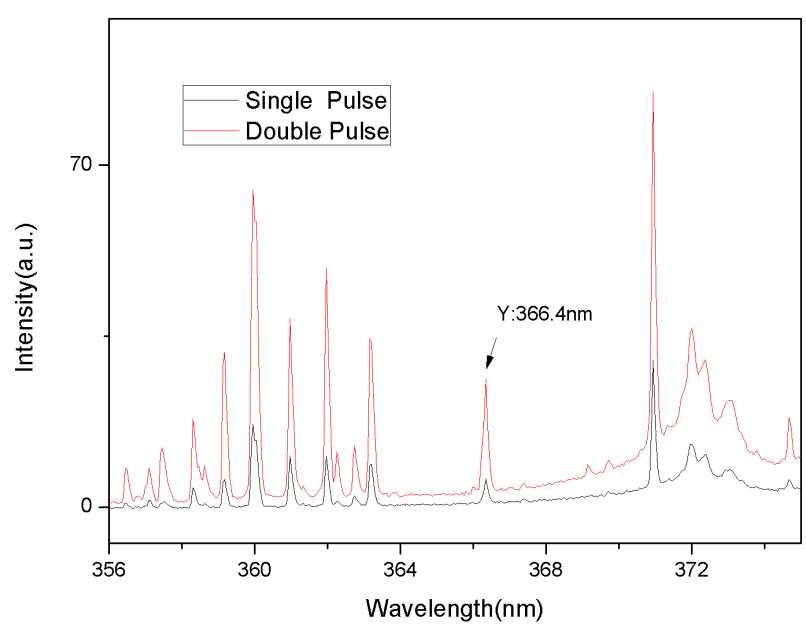

Figure 3. Comparison of DP-LIBS signalintensity with single pulse LIBS for Y (\#56).

the calibration curves, plotted as a function of the relative concentration in a linear scale. In Figure 4, the calibration curve of Y II shows a near straight line, it was calculated that the correlation coefficients of the ratio $\left(R^{2}\right)$ is 0.9998. Average relative error of calibration is less than 
$10 \%$ and the calibration curves that allow quantitative analysis in unknown samples [13]. In Figure 5, the calibration curve of $\mathrm{Zr} I$ is shown. The correlation coefficients of the ratio $\left(R^{2}\right)$ is 0.9547 , slightly poor compared with Y II at $366.4 \mathrm{~nm}$, which probably influenced by the spectral peak overlapped with other elements such as $\mathrm{Y}$, Gd, etc.

\section{Conclusion}

In this experiment, 5 magnesium alloy samples have been studied by a self-built remote DP-LIBS system. Compared with single pulse LIBS, the emission line intensities was enhanced about 4.7 times in DP-LIBS. The correlation coefficients of the calibration curves of Y II and Zr I were 0.9998 and 0.9547 respectively. The results of this study provide a potential DP-LIBS technique for metal smelting in real-time online chemical analysis and monitoring that used less energy to achieve enhanced spectra, and the distance between the system and targets

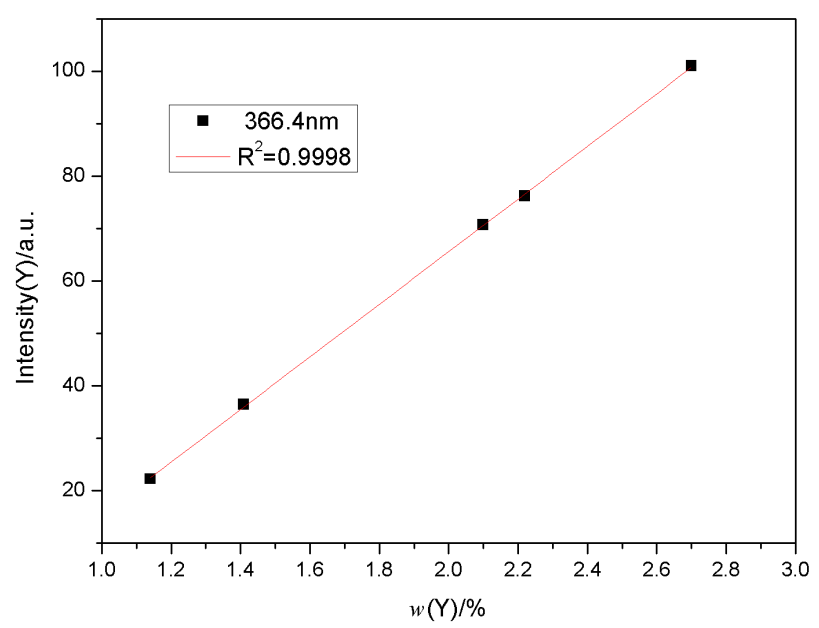

Figure 4. Calibration curve for Y II $366.4 \mathrm{~nm}$.

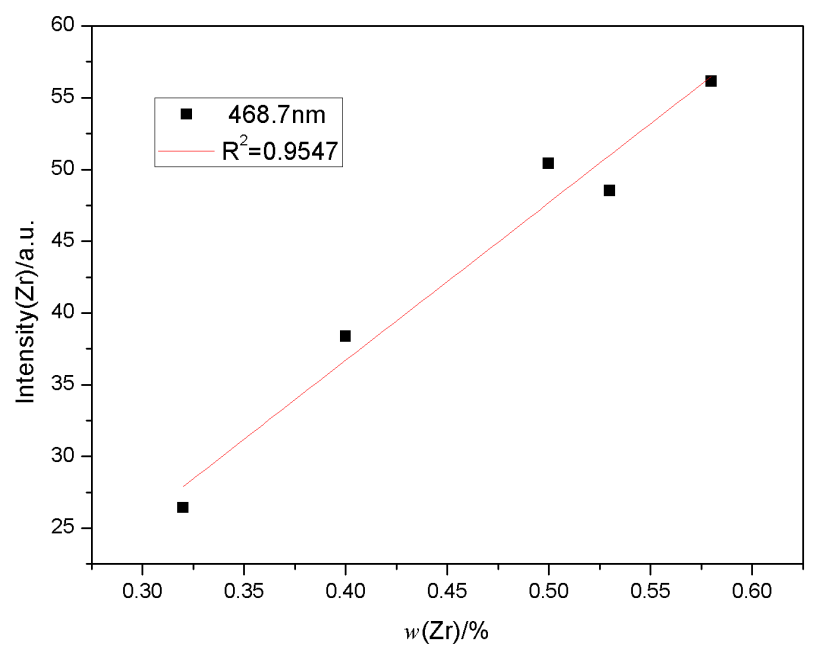

Figure 5. Calibration curve for $\mathrm{Zr} I 468.7 \mathrm{~nm}$. about $2.5 \mathrm{~m}$. The long-range detection distance of the LIBS system can be flexibility applied to the complex high-temperature environment of metal smelting process.

\section{Acknowledgements}

This work has been supported by the Equipment Development Programs of the Chinese Academy of Sciences (Grant No. YZ201247), the National High-Tech Research and Development Program of China (863 Program) (Grant No. 2012AA040608) and the National Natural Science Fund (Grant No. 61004131).

\section{REFERENCES}

[1] L. Radziemski and D. Cremers, "A Brief History of Laser-Induced Breakdown Spectroscopy: From the Concept of Atoms to LIBS 2012," Spectrochimica Acta Part B, Vol. 87, 2013, pp. 3-10.

http://dx.doi.org/10.1016/j.sab.2013.05.013

[2] B. Sallé, P. Mauchien and S. Maurice, "Laser-Induced Breakdown Spectroscopy in Open-Path Configurationfor the Analysis of Distant Objects," Spectrochimica Acta Part B, Vol. 62, 2007, pp. 739-768. http://dx.doi.org/10.1016/j.sab.2007.07.001

[3] G. Cristoforetti, S. Legnaioli, V. Palleschi, A. Salvetti and E. Tognoni, "Effect of Target Composition on the Emission Enhancement Observed in Double-Pulse Laser-Induced Breakdown Spectroscopy," Spectrochimica Acta Part B, Vol. 63, 2008, pp. 312-323. http://dx.doi.org/10.1016/j.sab.2007.11.006

[4] L. B. Guo, Z. Q. Hao, M. Shen, W. Xiong, X. N. He, Z. Q. Xie, M. Gao, X. Y. Li, X. Y. Zeng and Y. F. Lu, "Accuracy Improvement of Quantitative Analysisby Spatial Confinement in Laser-Induced Breakdown Spectroscopy,” OPTICS EXPRESS, Vol. 21, No. 15, 2013, pp. 1818818195. http://dx.doi.org/10.1364/OE.21.018188

[5] K. Rifai, F. Vidal, M. Chakera and M. Sabsabi, "Resonant Laser-Induced Breakdown Spectroscopy(RLIBS) Analysis of Traces through Selective Excitation of Aluminum in Aluminum Alloys," J. Anal. At. Spectrom, Vol. 28, 2013, pp. 388-395. http://dx.doi.org/10.1039/c3ja30308j

[6] N. B. Zorov, A. A. Gorbatenko, T. A. Labutin and A. M. Popov, "A Review of Normalization Techniques in Analytical Atomic Spectrometry with Laser Sampling: From Single to Multivariate Correction," Spectrochimica Acta Part B, Vol. 65, 2010, pp. 642-657. http://dx.doi.org/10.1016/j.sab.2010.04.009

[7] A. De Giacomoa, M. Dell'Aglio, F. Colao and R. Fantoni, "Double Pulse Laser Produced Plasma on Metallic Target in Seawater: Basic Aspects and Analytical Approach,” Spectrochimica Acta Part B, Vol. 59, 2004, pp. 14311438. http://dx.doi.org/10.1016/j.sab.2004.07.002

[8] V. I. Babushok, F. C. DeLucia Jr., J. L. Gottfried, C. A. Munson and A. W. Miziolek, "Double Pulse Laser Ablation and Plasma: Laser Induced Breakdown Spectroscopy Signal Enhancement," Spectrochimica Acta Part B, Vol. 61, 2006, pp. 999-1014. 
http://dx.doi.org/10.1016/j.sab.2006.09.003

[9] P. K. Diwakar, S. S. Harilal, J. R. Freeman and A. Hassanein, "Role of Laser Pre-pulse Wavelength and InterPulse Delay onSignal Enhancement in Collinear DoublePulse Laser-Induced Breakdown Spectroscopy," Spectrochimica Acta Part B, Vol. 87, 2013, pp. 65-73. http://dx.doi.org/10.1016/j.sab.2013.05.015

[10] Y. Lu, V. Zorba, X. Mao, R. Zheng and R. E. Russo, "UV fs-ns Double-Pulse Laser Induced Breakdown Spectroscopy for High Spatial Resolution Chemical Analysis,” $J$. Anal. At. Spectrom., Vol. 28, 2013, pp. 743-748. http://dx.doi.org/10.1039/c3ja30315b

[11] A. M. Matiaske, I. B. Gornushkin and U. Panne, "DoublePulse Laser-Induced Breakdown Spectroscopyfor Analysis of Molten Glass,” Anal Bioanal Chem, Vol. 402, 2012, pp. 2597-2606.

http://dx.doi.org/10.1007/s00216-011-5165-2

[12] J. L. Gottfried, F. C. De Lucia Jr., C. A. Munson and A. W. Miziolek, "Double-Pulse Standoff Laser-Induced Breakdown Spectroscopy for VersatileHazardous Materials Detection,” Spectrochimica Acta Part B, Vol. 62, 2007, pp. 1405-1411. http://dx.doi.org/10.1016/j.sab.2007.10.039

[13] V. K. Unnikrishnan, R. Nayak, P. Devangad, M. M. Tamboli, C. Santhosh, G. A. Kumar and D. K. Sardar, "Calibration Based Laser-Induced Break Down Spectroscopy (LIBS) for Quantitative Analysis of Doped Rare Earth Elements in Phosphors,” Materials Letters, Vol. 107, 2013, pp. 322-324.

http://dx.doi.org/10.1016/j.matlet.2013.06.036 